\title{
Effect of Mobile Phone Waves and Wi-Fi on Electrolytes and Oxidative Stress Indices of Saliva
}

${ }^{1}$ Ali Taghavi-Zonuz, ${ }^{2}$ Zahra Jamali, ${ }^{3}$ Solmaz Pourzare-Mehrbani, ${ }^{4}$ Mahdi Rahbar, ${ }^{5}$ Raana Tamgaji

\section{ABSTRACT}

Aim: The aim of this study is to investigate the effect of mobile phone waves and Wi-Fi on electrolytes and oxidative stress indices of saliva.

Materials and methods: A case group of $20 \mathrm{Wi}-\mathrm{Fi}$ and mobile phone users and a control group of 20 deaf people were selected. Saliva samples were obtained from both of these groups. Study variables, including the electrolytes and oxidative stress indices of saliva, were measured. The independent two-sample t-test was used to analyze the data. The statistical significance was defined at $p<0.05$.

Results: The mean sodium and malondialdehyde (MDA) levels in the case group were higher than in the control group. The results also showed that there was no significant difference between sodium and potassium levels ( $p=0.163$ and $p=0.892$, respectively) and the oxidative stress of total antioxidant (TAO) and MDA $(p=0.138$ and $p=0.115$, respectively) in either of the two groups. However, there was a significant difference in salivary nitric oxide (NO) levels $(p=0.04)$ between the case and control groups.

Conclusion: Electromagnetic waves affected the saliva composition to an extent that it caused a significant decrease in the NO levels of the case group.

Clinical significance: Nitric oxide acts as an active radical in nonspecific defense mechanisms in the oral cavity. So, reduction of $\mathrm{NO}$ with mobile phone and $\mathrm{Wi}-\mathrm{Fi}$ waves can cause disruption in the human defense system. These results illustrate the necessity of reducing contact with these waves.

Keywords: Electrolyte, Mobile phone, Oxidative stress, Saliva, Wi-Fi.

How to cite this article: Taghavi-Zonuz A, Jamali Z, PourzareMehrabani S, Rahbar M, Tamgaji R. Effect of Mobile Phone Waves and Wi-Fi on Electrolytes and Oxidative Stress Indices of Saliva. World J Dent 2017;8(5):370-373.

Source of support: This research was financially supported by the Research Council of the Tabriz University of Medical Sciences, Iran.

Conflict of interest: None

${ }^{1-3,5}$ Department of Oral Medicine, Faculty of Dentistry, Tabriz University of Medical Science, Tabriz, Islamic Republic of Iran

${ }^{4}$ Department of Operative and Esthetic Dentistry, Faculty of Dentistry, Tabriz University of Medical Science, Tabriz, Islamic Republic of Iran

Corresponding Author: Raana Tamgaji, Department of Oral Medicine, Faculty of Dentistry, Tabriz University of Medical Science, Tabriz, Islamic Republic of Iran, Phone: +09144060426 e-mail: R.tamgaji@gmail.com

\section{INTRODUCTION}

The use of mobile phones and Wi-Fi has increased considerably throughout the world. It is believed that $79 \%$ of Americans and more than $90 \%$ of European and Asian (including Iranian) teenagers have access to mobile phones. ${ }^{1-3}$ These devices are a source of nonionizing electromagnetic waves.

Several studies have investigated the effects of mobile phones on the oral cavity. Due to the special anatomic position of parotid glands in humans, there have been some specific studies on the effect of mobile phone waves on the volume of saliva, secretory changes, and their tumorigenicity. ${ }^{4}$ Hamzany et $a l^{4}$ stated that in mobile phone users, the amount secreted was higher, leading to a dilution of saliva. Subsequently, this decreased the total protein, albumin, and amylase levels and increased the level of oxidative stress indices. They suggested that this finding was a potential mechanism for increased salivary gland tumors in mobile phone users. In 2011, the International Agency for Research on Cancer, which is a part of the World Health Organization has categorized mobile phones as high risk Group 2B carcinogens (the possibility of causing cancers in humans according to limited evidence of studied material in carcinogenesis in human and insufficient evidence in animal). ${ }^{4,5}$ Some studies have investigated the simultaneous effects of mobile phones and Wi-Fi usage. One of these was the Yüksel et $\mathrm{al}^{6}$ study which showed that prolonged exposure to electromagnetic radiation by mobile phones and Wi-Fi decreases plasma prolactin, progesterone, and estrogen levels and increases uterine oxidative stress in rats. Nazıroğlu et $\mathrm{al}^{7}$ showed that oxidative stress due to exposure to Wi-Fi and mobile phones affects the reproductive male and female organs significantly. However, the above-mentioned studies were not done on saliva and, furthermore, it investigated the effects of simultaneous use of mobile phones and Wi-Fi in animals. In the current study, the oxidative stress indices including MDA, NO, and TAO have been studied in humans. In addition, the salivary electrolytes, mainly sodium and potassium levels, of both maximum and minimum users of Wi-Fi and mobile phone have been compared.

\section{MATERIALS AND METHODS}

A case group (maximum use) of 20 eligible people meeting the inclusion criteria, and attending in 2016, 
was selected from the Department of Oral Medicine, Faculty of Dentistry at Tabriz University of Medical Sciences. The interested persons were screened using the following inclusion criteria: At least 1 year history of mobile phone; at least half an hour daily talking on mobile phone; ${ }^{4}$ exposure to Wi-Fi at least 8 hours daily; ${ }^{7}$ minimum age of 18 .

Under the supervision of the Department of Social Welfare in Tabriz in Iran, a control group (minimum use) of 20 deaf persons was selected. The inclusion criteria for the control group were being deaf; minimum exposure to Wi-Fi (no use of Wi-Fi internet modem at home and/ or work place; minimum accidental exposure by routine attendance in public areas like airports and shops); and minimum age of 18 .

Exclusion criteria for both case and control groups were as follows: Use of hands-free or speaker for talking on mobile phones, pregnancy or breast feeding, history of any salivary gland disease such as xerostomia, consumption of any drug affecting saliva, tumor, radiotherapy, any chronic use, or abuse of medications, opioids, alcohol, and tobacco use.

The protocol for collecting the saliva samples from all the participants (both case and control) was as follows: Unstimulated saliva was collected at $10.00 \mathrm{AM}$ in a calm environment. Brushing of teeth, consumption of breakfast or any other food or drink, and physical activity had to be completed at least an hour and half before saliva collection. For a duration of 15 minutes, participants emptied their saliva into a container. The collected saliva was stored at $-70^{\circ} \mathrm{C}$ and then experimented for saliva electrolytes (sodium and potassium) by flame photometer (Model G, Series 11, USA), oxidative stress indices including MDA and NO, and TAO by spectrophotometer (PH 1016, Randox, Antrim, UK). ${ }^{4,8}$

For determining the normality or abnormality of data, the Kolmogorov-Smirnov test was used. Differences in the mean value of salivary potassium levels and oxidative stress indices between the two groups were analyzed by the Mann-Whitney U-test. The Statistical Package for the Social Sciences version 21 software for conducting independent two-sample t-test was used for comparing salivary sodium levels. The statistical significance was set at $\mathrm{p}<0.05$.

\section{FINDINGS}

Statistical analysis showed that there was no significant difference between sodium and potassium levels ( $p=0.163$ and $p=0.892$, respectively) and oxidative stress of TAO and MDA ( $p=0.138$ and $p=0.115$, respectively) in both case and control groups. However, there was a significant difference between the salivary $\mathrm{NO}$ levels of the two groups $(\mathrm{p}=0.04)$ (Table 1$)$.
Table 1: Mean value and standard deviation of salivary electrolytes and oxidative stress indices in case and control groups

\begin{tabular}{llll}
\hline & & Control group & Case group \\
\hline Salivary electrolytes & Sodium & $4.1 \pm 43.85$ & $6 \pm 45.5$ \\
& Potassium & $5.7 \pm 10.7$ & $4.11 \pm 10,44$ \\
Salivary oxidative & TAO & $0.52 \pm 1.3$ & $0.57 \pm 0.86$ \\
stress indices & MDA & $0.17 \pm 1.08$ & $0.32 \pm 1.22$ \\
& NO & $198 \pm 384$ & $258 \pm 169$ \\
\hline
\end{tabular}

\section{DISCUSSION}

The use of Wi-Fi and mobile phones is increasing rapidly in schools, workplaces, and homes. ${ }^{7}$ The widespread use of such devices causes an increase in the electromagnetic resonance (EMR). The biological effect of EMR and its consequences is a challenging discussion. In reference to the current study, $20 \mathrm{Wi}-\mathrm{Fi}$ and mobile phone users and 20 deaf people were selected as case group and control group respectively. Saliva samples were collected from both groups. Statistical analysis showed that there was no significant difference between sodium and potassium levels and oxidative stress indices of TAO and MDA in either the case or control groups. However, the difference between salivary NO levels in case and control groups was significant $(\mathrm{p}=0.04)$.

Studies show that salivary potassium and chloride levels have a significant relationship with active dental caries. ${ }^{8}$ The functions of sodium include body fluid control, balancing the acid and base levels $(\mathrm{pH})$, neural conduction, and blood pressure control..$^{9}$ Statistical analysis showed that there was no significant difference between sodium and potassium levels in either the case or control groups. There were no other similar studies to compare the results.

Oxidative stress induces lipid peroxidation, which leads to production of huge amount of chemicals like MDA. Previous studies showed that MDA has an important role in carcinogenesis as it interacts with the deoxyribonucleic acid (DNA) and produces extra DNA-MDA. ${ }^{10}$ An increase in lipid peroxidation and MDA causes a significant destruction in the red blood cell membrane, which is also rich in unsaturated fatty acids. The increase in free radical levels increases lipid peroxidation too. In other words, the compensatory increase in oxidative stress is followed by a decrease in antioxidant levels. ${ }^{11}$

Statistical analysis showed that there was no significant difference between oxidative stress of TAO and MDA levels in either the case or control groups. A study conducted by Manifar et $\mathrm{al}^{12}$ on the changes in lipid peroxidation and TAO in head and neck cancer patients showed that the level of these variables increased following radiotherapy. The lipid peroxidation increased a bit in head and neck cancer patients but the MDA level 
which is a lipid peroxidation marker decreased. As the Manifar study has investigated the effects of X-rays, the results are different from the current one which studies the effect of electromagnetic waves.

A study was conducted by Atasoy on the effects of Wi-Fi in rats. This study showed that when rats were exposed to $2.45 \mathrm{GHz}$ waves for 4 weeks, oxidative stress decreased. However, when exposed to electromagnetic waves in the range of 900 to $1800 \mathrm{GHz}$, the antioxidant level increased in comparison to the control group. ${ }^{13}$ Although these findings on oxidative stress are consistent with the current study, the antioxidant level findings between the two studies are not compatible. The reasons for this conflict could be varied. For instance, the usage time or frequency of exposure to electromagnetic waves could have a dissimilar or a new effect on saliva composition. The discrepancies in the results of current study vis-à-vis Atasoy study could also be an outcome of the fact that the latter was an animal study and was performed in a specific frequency and time range.

In contrast to the current study, Nazıroğlu et $\mathrm{al}^{7}$ reported a relationship between electromagnetic wave exposure and oxidative stress levels. A study by ArbabiKalati et $\mathrm{al}^{14}$ on the effect of duration of mobile phones usage on the salivary TAO level and salivary immunoglobulin A showed that the mean value of TAOs has a significant relationship with exposure to mobile phone waves. Total antioxidant levels of people with longer daily exposure to electromagnetic waves are lower than those users who were exposed to shorter daily durations of electromagnetic waves. The study of Khalil et al ${ }_{1}^{15}$ with the purpose of evaluating salivary oxidant and antioxidant levels in mobile phone users, showed that short-term use of mobile phones did not have any effect on oxidative stress indices and antioxidants. The results of this study showed that MDA level increased with increase in mobile phone usage time and had its maximum effect in 30 minutes; however, a significant relationship was not found. The mobile phone model and the method of saliva sample collection (whether collected immediately after talking or not) could influence the saliva composition. Furthermore, the Arbabi-Kalati et a ${ }^{14}$ study did not have a control group for comparison. All these reasons could contribute to the resultant discrepancies between this mentioned study and the current one.

Nitric oxide is a signaling molecule that acts as a double-edged sword and affects many physiologic and pathologic processes. Low levels of NO are related to homeostatic processes like immunologic activities, blood flow, platelet aggregation, neural conduction, and memory. Increased production of NO is seen in inflammatory and immunologic disease, pain, neurologic disorders, arthrosclerosis, and cancer. ${ }^{16,17}$
Nitric oxide acts as an active radical in nonspecific defense mechanisms in the oral cavity. It is believed that the antibacterial effect of $\mathrm{NO}$ is expressed in two ways: Prevention of bacterial growth and increase in cytotoxicity by salivary macrophages. ${ }^{18}$ Senthil et a ${ }^{19}$ showed that low levels of caries in children is related to increase in $\mathrm{NO}$ levels. Metgud et $\mathrm{al}^{20}$ showed that extreme increase in $\mathrm{NO}$ can have a destructive and inflammatory effect in the oral cavity. For example, oral lichen planus is a chronic inflammatory process in which NO produced by L-arginine amino acid by NO synthase enzyme has an important role. The results of the current study show that the NO levels decreased significantly in the case group. No study was found in reference to the effect of electromagnetic waves on NO levels in saliva.

An evaluation based on age, sex, duration of time exposed to electromagnetic waves, and usage of handsfree talking on the phone (which was an exclusion criteria in the current study) is suggested for further studies.

\section{CONCLUSION}

There was no significant difference between sodium or potassium levels and the oxidative stress of TAO and MDA in either the case or control groups. The NO level was significantly lower in the case group.

\section{REFERENCES}

1. Davis DL, Miller AB, Philips A. Association of mobile phone use with adult brain cancer remains plausible. Br Med J 2012;344:e3083.

2. Hardell L, Carlberg M, Söderqvist F, Mild KH, Morgan LL. Long-term use of cellular phones and brain tumours: increased risk associated with use for 210 years. J Occup Environ Med 2007; 64(9): 626-632.

3. Janssens JP. Mobile phones and cancer? Eur J Canc Prev 2005;14:81-82.

4. Hamzany Y, Feinmesser R, Shpitzer T, Mizrachi A, Hilly O, Hod R, Bahar G, Otradnov I, Gavish M, Nagler RM. Is human saliva an indicator of the adverse health effects of using mobile phones? Antioxidants Redox Signal 2013; 18(6): 622-627.

5. Simard EP, Torre LA, Jemal A. International trends in head and neck cancer incidence rates: differences by country, sex and anatomic site. Oral Oncol 2014 May;50(5):387-403.

6. Yüksel M, Nazıroğlu M, Özkaya MO. Long-term exposure to electromagnetic radiation from mobile phones and $\mathrm{Wi}-\mathrm{Fi}$ devices decreases plasma prolactin, progesterone, and estrogen levels but increases uterine oxidative stress in pregnant rats and their offspring. Endocrine 2016 May;52(2):352-362.

7. Nazıroğlu M, Yüksel M, Köse SA, Özkaya MO. Recent reports of Wi-Fi and mobile phone-induced radiation on oxidative stress and reproductive signaling pathways in females and males. J Membr Biol 2013 Dec;246(12):869-875.

8. Dodds MW, Johnson DA, Mobley CC, Hattaway KM. Parotid saliva protein profiles in caries-free and caries-active adults. Oral Surg Oral Med Oral Pathol Oral Radiol Endod 1997 Feb;83(2):244-251. 
9. Afridi HI, Kazi TG, Kazi N, Jamali MK, Arain MB, Jalbani N, Sarfaraz RA, Shah A, Kandhro GA, Shah AQ, et al. Potassium, calcium, magnesium, and sodium levels in biological samples of hypertensive and nonhypertensive diabetes mellitus patients. Biol Trace Elem Res 2008 Sep;124(3): 206-224.

10. Blair IA. DNA adducts with lipid peroxidation products. J Biol Chem 2008 Jun 6; 283(23): 15545-15549.

11. Halliwell B. Oxidative stress and cancer: have we moved forward? Biochem J 2007 Jan 1;401(1): 1-11.

12. Manifar S, Abbassi F, Dizgah IM, Khatami R, Esmseil M, Almasi A. Alteration of lipid peroxidation and total antioxidant capacity in patients with head and neck cancers following radiotherapy. J Arch Mil Med 2015;3(4).

13. Atasoy HI, Gunal MY, Atasoy P, Elgun S, Bugdayci G. Immunohistopathologic demonstration of deleterious effects on growing rat testes of radiofrequency waves emitted from conventional Wi-Fi devices. J Pediatr Urol 2013 Apr;9(2): 223-229.

14. Arbabi-Kalati F, Salimi S, Vaziry-Rabiee A, Noraeei M. Effect of mobile phone usage time on total antioxidant capacity of saliva and salivary immunoglobulin A. Iran J Public Health 2014 Apr;43(4):480-484.
15. Khalil AM, Abu Khadra KM, Aljaberi AM, Gagaa MH, Issa HS. Assessment of oxidant/antioxidant status in saliva of cell phone users. Electromagn Biol Med 2014 Jun;33(2):92-97.

16. Tvarijonaviciute A, Aznar-Cayuela C, Rubio CP, Ceron JJ, LópezJornet P. Evaluation of salivary nitric oxide levels and C-reactive protein in patients with oral lichen planus and burning mouth syndrome. J Oral Pathol Med 2017 May;46(5):387-392.

17. Mehdipour M, Taghavi Zenouz A, Bahramian A, Gholizadeh N, Boorghani M. Evaluation of serum nitric oxide level in patients with oral lichen planus. J Dent (Shiraz) 2014 Jun;15(2):48-51.

18. Kasapović J, Pejić S, Stojiljković V, Todorović A, RadoševićJelić L, Saičić ZS, Pajović SB. Antioxidant status and lipid peroxidation in the blood of breast cancer patients of different ages after chemotherapy with 5-fluorouracil, doxorubicin and cyclophosphamide. Clin Biochem 2010 Nov;43(16-17): 1287-1293.

19. Senthil Eagappan AR, Rao VA, Sujatha S, Senthil D, Sathiyajeeva J, Rajaraman G. Evaluation of salivary nitric oxide level in children with early childhood caries. Dent Res J 2016 Jul-Aug;13(4):338-341.

20. Metgud R, Anandani C, Singh K. Estimation of salivary nitric oxide in oral precancer patients. Biotech Histochem 2015 May;90(4):302-308. 